\title{
Metabolic engineering strategies to produce medium-chain oleochemicals via acyl-ACP:CoA transacylase activity
}

\section{Qiang Yan}

University of Wisconsin-Madison

\section{William Cordell}

University of Wisconsin-Madison

\section{Michael Jindra}

University of Wisconsin-Madison

\section{Dylan Courtney}

University of Wisconsin-Madison

\section{Madeline Kuckuk}

University of Wisconsin-Madison

\section{Xuanqi Chen}

University of Wisconsin-Madison

\section{Brian Pfleger ( $\nabla$ brian.pfleger@wisc.edu )}

University of Wisconsin-Madison https://orcid.org/0000-0002-9232-9959

\section{Article}

Keywords: Oleochemical, PhaG, 3-hydroxyacyl-ACP:CoA transacylase, fatty acid, fatty alcohol, methyl ketone, poly-hydroxyalkanoate

Posted Date: August 27th, 2021

DOl: https://doi.org/10.21203/rs.3.rs-842403/v1

License: (a) (1) This work is licensed under a Creative Commons Attribution 4.0 International License. Read Full License

Version of Record: A version of this preprint was published at Nature Communications on March 25th, 2022. See the published version at https://doi.org/10.1038/s41467-022-29218-3. 


\title{
Metabolic engineering strategies to produce medium-chain oleochemicals via acyl-ACP:CoA transacylase activity
}

\author{
Qiang Yan, ${ }^{\mathrm{a}, \mathrm{b}}$, William T. Cordell ${ }^{\mathrm{a}, \mathrm{b}}$, Michael A. Jindra ${ }^{\mathrm{a}, \mathrm{b}}$, Dylan Courtney ${ }^{\mathrm{a}}$, Madeline K \\ Kuckuk $^{\mathrm{a}}$, Xuanqi Chen ${ }^{\mathrm{a}}$, and Brian F. Pfleger ${ }^{\mathrm{a}, \mathrm{b}, \mathrm{c}, *}$
}

a Department of Chemical and Biological Engineering, University of Wisconsin-Madison,

Madison, WI 53706, United States

b DOE Center for Advanced Bioenergy and Bioproducts Innovation, University of

Wisconsin-Madison, Madison, WI 53706, United States

$\mathrm{c}$ Microbiology Doctoral Training Program, University of Wisconsin-Madison, Madison, WI 53706, United States

* Corresponding author. 3629 Engineering Hall, 1415 Engineering Drive, Madison, WI 53706, United States. Phone: $+1608 \quad 890$ 1940. Fax: +1608 262-5434. E-mail address: pfleger@engr.wisc.edu. 


\begin{abstract}
Microbial lipid metabolism is an attractive route for producing aliphatic chemicals, commonly referred to as oleochemicals. The predominant metabolic engineering strategy centers on heterologous thioesterases capable of producing fatty acids of desired size. To convert acids to desired oleochemicals (e.g. fatty alcohols, ketones), metabolic engineers modify cells to block beta-oxidation, reactivate fatty acids as coenzyme-A thioesters, and redirect flux towards termination enzymes with broad substrate utilization ability. These genetic modifications narrow the substrate pool available for the termination enzyme but cost one ATP per reactivation - an expense that could be saved if the acyl-chain was directly transferred from ACP- to CoA-thioester. In this work, we demonstrate an alternative acyl-transferase strategy for producing medium-chain oleochemicals. Through bioprospecting, mutagenesis, and metabolic engineering, we developed strains of Escherichia coli capable of producing over $1 \mathrm{~g} / \mathrm{L}$ of medium-chain free fatty acids, fatty alcohols, and methyl ketones using the transacylase strategy.
\end{abstract}




\section{HIGHLIGHTS}

- Validated PhaG as a 3-hydroxyacyl-ACP:CoA transacylase with in vivo characterization

- Bioprospected a higher activity PhaG homolog from Pseudomonas koreensis

- Isolated a PhaG Q45R G142V variant that had a 4-fold improvement in fatty acid production when compared to wild-type

- We reported production of fatty acid and fatty alcohol over $1 \mathrm{~g} / \mathrm{L}$, and methyl ketone 7.2 $\mathrm{g} / \mathrm{L}$

\section{Keywords}

Oleochemical; PhaG; 3-hydroxyacyl-ACP:CoA transacylase; fatty acid; fatty alcohol; methyl ketone; poly-hydroxyalkanoate 


\section{INTRODUCTION}

Oleochemicals are a class of aliphatic hydrocarbons that are industrially-derived from petroleum, animal fats or oil seed crops. Depending on the terminal reductive state of the acylchain, oleochemicals can be classified as fatty acids, alcohols, aldehydes, ketones, esters, olefins, alkanes, etc. Oleochemicals are also categorized by size (i.e. acyl chain length). Medium-chain oleochemicals are defined as compounds with acyl moiety of 8-12 carbons and are of particular interest due to their applications as commodity and specialty chemicals ${ }^{1,2}$. For example, mediumchain fatty acids are used as herbicides, antimicrobials, and precursors for lubricant synthesis ${ }^{3}$. Medium-chain methyl ketones are used as flavors, floral fragrances, insecticides, and renewable liquid transportation fuels ${ }^{4,5}$. Medium-chain alcohols are used as surfactants, as additives in biodiesel, as cosmetics and in consumer products. The unique molecular and chemical properties of medium-chain oleochemicals and their limited natural abundance make them attractive targets for biosynthesis.

The final enzymatic reactions in oleochemical biosynthesis use acyl-thioesters as substrates, making high-flux to desired chain length acyl-thioesters a key element to any metabolic engineering strategy. Thus far, two approaches have been applied to produce medium-chain acylthioesters in microbes: thiolase-driven $\beta$-reduction cycles (i.e. reversal of $\beta$-oxidation) ${ }^{6}$ and thioesterase-driven diversion of fatty acid biosynthesis intermediates ${ }^{1,7}$. The reverse $\beta$-oxidation pathway incorporates a reversible, thiolase-catalyzed Claisen condensation (e.g. FadA) with acetyl-CoA as the donor to by-pass the energy consumption required for producing malonyl-CoA in fatty acid biosynthesis. The 1-ATP savings per elongation provides reversal of $\beta$-oxidation with the highest theoretical yield of all oleochemical biosynthesis routes at the cost of losing the strong driving force provided by the decarboxylative Claisen condensation in fatty acid biosynthesis. $\beta$ - 
reduction was used to demonstrate production of both medium-chain fatty acids and fatty alcohols $^{6,8-11}$ in high yields, albeit with poor selectivity to specific chain length products. This is in part due to the competition between termination reactions (cleavage or reduction, respectively) and thiolase-catalyzed extension of acyl-CoA thioesters. To date, strategies to bias termination at a particular chain length have yet to be demonstrated and better results have come from finding ways to accumulate pools of desired chain-length acyl-CoAs made via fatty acid biosynthesis to avoid the competition entirely.

Plants synthesize novel oils containing medium-chain acyl groups by expressing specific acyl-ACP thioesterases in the chloroplast to generate free fatty acids of desired size ${ }^{7}$. Once made, the free fatty acids are transported to the cytosol for reactivation as acyl-CoA and subsequent incorporation into storage lipids ${ }^{12}$. In microbes, plant acyl-ACP thioesterases can be leveraged to produce free fatty acids of desired chain length. Additional genetic modifications are needed to enable conversion of the free fatty acids to the desired oleochemical form (e.g. alcohol, ketone, ester). Unlike plants where biochemistries can be compartmentalized, bacteria express enzymes for both fatty acid biosynthesis and catabolism in the same locations. In order to accumulate a pool of desired acyl-CoA's $\beta$-oxidation must be blocked by eliminating all enzymes that catalyze one of the four reactions in the cycle. Depending on the desired product form, different $\beta$-oxidation steps are targeted (e.g. acyl-CoA dehydrogenase, FadE, for fatty alcohol production; thiolase, FadA/FadI, for methyl ketone production) to produce the substrate for termination enzymes (e.g. saturated acyl-CoA for acyl-CoA reductase conversion to fatty alcohol; $\beta$-ketoacyl-CoA for $\beta$ ketothioesterase conversion to methyl ketone). The last steps in the metabolic engineering strategy are expression of a specialized acyl-ACP thioesterase (to produce desired free fatty acids), expression of an acyl-CoA synthetase (to activate the free fatty acid), and expression of the desired 
termination enzyme. Highly-active acyl-ACP thioesterases have been identified from natural sources and others have been engineered or evolved in the laboratory to produce octanoic acid, decanoic acid, dodecanoic acid, and tetradecanoic acid ${ }^{13-17}$. For instance, a highly active variant of a Cuphea palustris thioesterase FatB (referred to ${ }^{C p} \mathrm{FatB}{ }^{*}$ ) was identified using a ${ }^{C p} \mathrm{FatB}$ random mutagenesis library and a growth selection based on the lipoic acid requirement of Escherichia coli. E. coli strain NHL17 (MG1655 $\triangle a r a B A D \triangle f a d D:: t r c{ }^{C p} f a t B^{*}$ ) produced $1.7 \mathrm{~g} / \mathrm{L}$ octanoic acid with $>90 \%$ specificity from $20 \mathrm{~g} / \mathrm{L}$ glycerol ${ }^{14}$. The highly-active $\mathrm{C} 8$-specific ${ }^{C p}$ FatB* enzyme was utilized to produce 1-octanol by expressing an acyl-CoA synthetase ( ${ }^{M t} \mathrm{FadD6}$ ) from Mycobacterium tuberculosis to reactivate octanoic acid at the cost of 1 mole ATP and expressing an acyl-CoA reductase from Marinobacter aquaeolei $\left({ }^{\mathrm{Ma}} \mathrm{ACR}\right)$ to convert octanoyl-CoA to 1octanol. The resulting E. coli strain NHL24 produced $1.3 \mathrm{~g} / \mathrm{L} 1$-octanol ${ }^{18}$. In a separate study, 2heptanone was produced by converting octanoyl-CoA to $\beta$-ketooctanoyl-CoA via an acyl-CoA oxidase from Micrococcus luteus (Mlut_11700) and an endogenous bi-functional dehydrogenase ${ }^{E c}$ FadB. Subsequently, $\beta$-ketooctanoyl-CoA was hydrolyzed by an $\beta$-ketoacyl-CoA thioesterase ${ }^{P_{s}}$ FadM from Providencia sneebia $\left({ }^{P_{s}} \mathrm{FadM}\right)$ and the resulting $\beta$-ketooctanoic acid was decarboxylated non-enzymatically to yield 2-heptanone. Strain E. coli TRS12 (MG1655 $\triangle$ araBAD $\triangle f a d D: \because P t r c-{ }^{C P} f a t B 1 * \Delta f a d A \quad \Delta f a d E \quad \Delta f a d I \quad \Delta f a d R$ ) harboring pTRC99a- ${ }^{M t} f a d D 6-{ }^{P s} f a d M$ and pACYC- ${ }^{M l}{ }^{m} l u t$ - 11700 plasmids produced up to $4 \mathrm{~g} / \mathrm{L}$ 2-heptanone in fed-batch bioreactor experiments ${ }^{4}$. While successful, this thioesterase strategy could be further improved by replacing the futile cycle of thioester cleavage and formation with a direct acyl-transfer.

In nature, some bacteria such as Pseudomonads, accumulate polyhydroxyalkanoate (PHA) as a means of storing carbon and energy. PHA polymerization requires a supply of $(R)-3-$ hydroxyacyl-CoAs (PHA monomers), which can be derived from either fatty acid biosynthesis or 
$\beta$-oxidation. Two decades ago, PhaG, an enzyme found in Pseudomonas putida and Pseudomonas aeruginosa, was identified as the enzymatic link between fatty acid biosynthesis and PHA biosynthesis ${ }^{19,20}$. PhaG was hypothesized to catalyze the transfer of the $(R)$-3-hydroxyacyl moiety from the $\mathrm{ACP}$ thioester to $\mathrm{CoA}$. In vitro experimental results showed a time-course of CoA release by incubating purified $\mathrm{PhaG},(R)-3$-hydroxydecanoyl-CoA and holo-ACP, indicating $\mathrm{PhaG}$ catalyzes a reversible tranacylase reaction ${ }^{21}$. In subsequent studies, researchers overexpressed PhaG and observed increased PHA content in cells and an increased fraction of medium-chainlength 3-hydroxyalkanoate (3HA) units in the polymer ${ }^{22-25}$. Given these results, we hypothesized that PhaG could provide a similar role in linking fatty acid biosynthesis with creation of tailored pools of acyl-CoAs and oleochemical products.

In the present study, we demonstrated that such a PhaG-dependent pathway is an alternative strategy to link fatty acid biosynthesis and oleochemical production with higher theoretical yields that previously demonstrated thioesterase strategies (Figure 1 and Table S2). We validated the ability of $P$. putida $\mathrm{PhaG}$, to perform 3-hydroxyacyl-ACP:CoA transacylase reactions and proved that $\mathrm{PhaG}$ can direct flux towards oleochemical synthesis at rates comparable to thioestereases. Using computational bioprospecting tools, we identified seven homologs of ${ }^{P p u} \mathrm{PhaG}$ and evaluated their in vivo activities. The PhaG variant from Pseudomonas koreensis produced 1.6-fold more methyl ketones than the ${ }^{P p u} \mathrm{PhaG}$ variant used as a seed to the search. We constructed a random mutagenesis library of ${ }^{P k} \mathrm{PhaG}$ and isolated seventeen beneficial mutations that increased octanoic acid production 3.3- to 16.3-fold above strains expressing the original ${ }^{P k} \mathrm{PhaG}$. We used these improved enzymes to construct strains capable of producing three demonstration oleochemicals - free fatty acids, fatty alcohols, and methyl ketones. Strains expressing the PhaG-dependent pathway were capable of producing $1.1 \mathrm{~g} / \mathrm{L} \mathrm{C} \mathrm{C}_{8}-\mathrm{C}_{14}$ free fatty acids, 
$1.5 \mathrm{~g} / \mathrm{L} \mathrm{C} \mathrm{C}_{7}-\mathrm{C}_{15}$ methyl ketones, and $1.1 \mathrm{~g} / \mathrm{L} \mathrm{C}_{8}-\mathrm{C}_{16}$ fatty alcohols depending on the tailoring enzymes co-expressed. These titers demonstrate that PhaG is a useful alternative for medium-chain length oleochemical synthesis and a promising target for future protein engineering to guide substrate selectivity. 


\section{MATERIALS AND METHODS}

\subsection{Bacterial strains, plasmids, oligonucleotides, and reagents}

All bacterial strains used in this study are listed in Supplementary Table S1. Q5 DNA polymerase and Monarch $^{\circledR}$ PCR and DNA Cleanup Kit was purchased from New England Biolabs (Ipswich, MA). Oligonucleotide primers and gBlocks were synthesized by Integrated DNA Technologies (IDT), Inc. (San Diego, CA). Chemicals including fatty acid, fatty alcohol and methyl ketones were purchased from Sigma-Aldrich (St. Louis, MO).

E. coli DH5 $\alpha$ strains were used for plasmid amplification and DNA assembly. E. coli RABIJ and E. coli CM23 were created as part of prior studies ${ }^{11,26}$. E.coli RAI and RADI strains were used in those experiments presented in Figure 2B, 2C, 2D, 3B, 5A, 5B, 6A and 6B. E. coli CM23 strains were used in those experiments presented in Figure $\mathbf{4 B}$ and $\mathbf{4 C}$.

\subsection{Plasmid and strain construction}

All plasmids used in this study are summarized in Supplementary Table S1. Plasmid maps are available as Supplementary Materials. All plasmids were constructed by Gibson assembly (New England Biolabs). The chromosomal deletion of $f a d D$ and lipB were performed using a combination of lambda red recombination and CRISPR/Cas9-mediated selection as described in prior work 4,11,14,18. All cloned sequences and gene deletions were confirmed by Sanger sequencing performed by Functional Biosciences (Madison, WI). Constructs expressing FadB and PhaJ homologs were obtained from prior studies ${ }^{4,11,26}$. 


\subsection{Oleochemical production and quantification}

All oleochemical production studies were performed by growing E. coli strains at $30^{\circ} \mathrm{C}$ in Clomburg medium containing $20 \mathrm{~g} / \mathrm{L}$ glycerol, the appropriate antibiotics (Carbenicillin - 100 $\mu \mathrm{g} / \mathrm{mL}$; kanamycin - $50 \mu \mathrm{g} / \mathrm{mL}$; chloramphenicol - $34 \mu \mathrm{g} / \mathrm{mL})$, and IPTG for induction as indicated. Pre-cultures for each experiment were prepared by inoculating $5 \mathrm{~mL}$ LB media (+ antibiotics) with a single colony and incubating overnight at $30^{\circ} \mathrm{C}$ with shaking at 250 r.p.m. A 2.5 vol\% inoculum was transferred into production flasks with a starting $\mathrm{OD}_{600} 0.1$. Fatty alcohol and methyl ketone production cultures were supplemented with $10 \%(\mathrm{v} / \mathrm{v})$ dodecane to provide a product sink. Samples from each culture were extracted after 72 hours incubation at $30^{\circ} \mathrm{C}$.

Fed-batch fermentation was performed using a 1-L Infors Multifors bioreactor. Overnight pre-cultures were inoculated to an initial $\mathrm{OD}_{600}$ of 0.05 into a bioreactor containing $500 \mathrm{~mL}$ Clomburg medium with $\sim 50 \mathrm{~g} / \mathrm{L}$ glycerol. The bioreactor was operated at the following conditions: temperature was controlled at $30^{\circ} \mathrm{C}$ post-induction, air flow was $1.5 \mathrm{~L} / \mathrm{min}$, stirrer rate was varied between 250 r.p.m. and 1000 r.p.m. to control dissolved oxygen at a value of 30\%, pH was maintained at 7.0 using $2 \mathrm{M}$ sulfuric acid and $2 \mathrm{M}$ ammonia hydroxide. When the $\mathrm{OD}_{600}$ reached 1.0, IPTG was added to achieve a final concentration of $1 \mathrm{mM}$ and $100 \mathrm{~mL}$ dodecane was fed in the bioreactor. At 24 hours of post-induction, $\sim 100 \mathrm{~mL} 5 \mathrm{X}$ concentrated Clomburg media containing $500 \mathrm{~g} / \mathrm{L}$ glycerol was one-time bolus-fed into the bioreactor and fermentation terminated 96 hours post-induction. Measurements of methyl ketone, glycerol, optical density, and $\mathrm{CO}_{2}$ evolution were recorded for 96 hours total.

The bioreactor outlet gas stream was directed through a chilled organic absorber to capture methyl ketone vapors stripped from the culture broth. The absorber was composed of a jacketed glass gas dryer with a ceramic sparge distributing the off gas into $100 \mathrm{~mL}$ of chilled dodecane. The 
temperature of the absorber was maintained at $5^{\circ} \mathrm{C}$ by an external water cooler. Methyl ketone data was taken at 96 hours from the dodecane, and endpoint methyl ketone capture was taken from both the collected water and remaining dodecane 96-101 hours after inducing the culture. A schematic of the absorber is described in more detail in Supplemental Figure S6.

Methods of fatty acid, fatty alcohol and methyl ketone quantification were described in previous work ${ }^{4}$. To determine the methyl ketone and fatty alcohol concentration in the distinct organic or aqueous phases, $50 \mathrm{~mL}$ of cell culture was centrifuged at $4500 \times \mathrm{g}$ for $10 \mathrm{~min}$ and 0.5 $\mathrm{mL}$ samples from the dodecane layer and $2.5 \mathrm{~mL}$ samples from aqueous phase were collected and evaluated separately. Fatty acids were extracted from culture according to an acid-based esterification method described previously ${ }^{14,15}$. Fatty alcohols and methyl ketones were extracted from culture into $n$-hexane according to methods described previously ${ }^{4,11}$. Fatty acid and methyl ketone species were separated using an Agilent RTX-5 column and fatty alcohol species were separated using Agilent DB-Fatwax column (Santa Clara, CA). Oleochemicals were quantified by comparing GC-FID peak areas against standard curves prepared with commercial standards.

\subsection{Mutagenesis of PhaG}

A mutagenic PhaG library was constructed by error-prone PCR using GeneMorph II from Agilent (Senta Clara, CA) with a low mutation frequency (0 to 4.5 mutations $/ \mathrm{kb}$ ). The plasmid backbone (pBTRCK) was PCR-amplified using a high-fidelity DNA polymerase Q5 from New England Biolabs (Ipswich, MA). The library was assembled using an isothermal assembly method ${ }^{27}$. Primers used in the creation of the library contained the start and stop codons in order to prevent mutation of them. 
The sequence of improved PhaG variants was obtained by Sanger sequencing of colony PCR products made by high fidelity Q5 DNA polymerase. To re-introduce single point mutations, we amplified ${ }^{P k} \mathrm{PhaG}$ with mutagenic primers and subcloned the fragments into pTRC99A- ${ }^{P k} p h a G{ }^{\prime}{ }^{\text {Td }}$ TER by an isothermal assembly method ${ }^{27}$. The resulting plasmids were transformed into E. coli CM23 harboring pBTRCK- ${ }^{M a}$ tes $B^{*}$ and pACYC- ${ }^{P a} p h a J 3$ plasmids.

\subsection{Lipoic acid selection}

3-Hydroxyoctanoyl-CoA producing variants of PhaG were isolated using a lipoic/octanoic acid selection described previously ${ }^{14}$. Plasmids expressing each variant (pBTRCK- ${ }^{P k} p h a G^{*}$ ) were transformed into E. coli CM23-4lipB (CM23 strain, AlipB) strain harboring pTRC99a- ${ }^{T d}$ TER${ }^{M a}$ tes $B^{*}+$ pACYC- ${ }^{P a} p h a J 3$. Transformants were plated on MOPS minimal media agarose plates containing $0.2 \%$ glucose, $20 \mu \mathrm{M}$ IPTG, and kanamycin, chloramphenicol and carbenicillin to maintain and induce plasmids as described in previous work ${ }^{14}$. Transformants were patched onto LB plates for archiving and secondary screening in octanoic acid production studies. 


\section{RESULTS AND DISCUSSION}

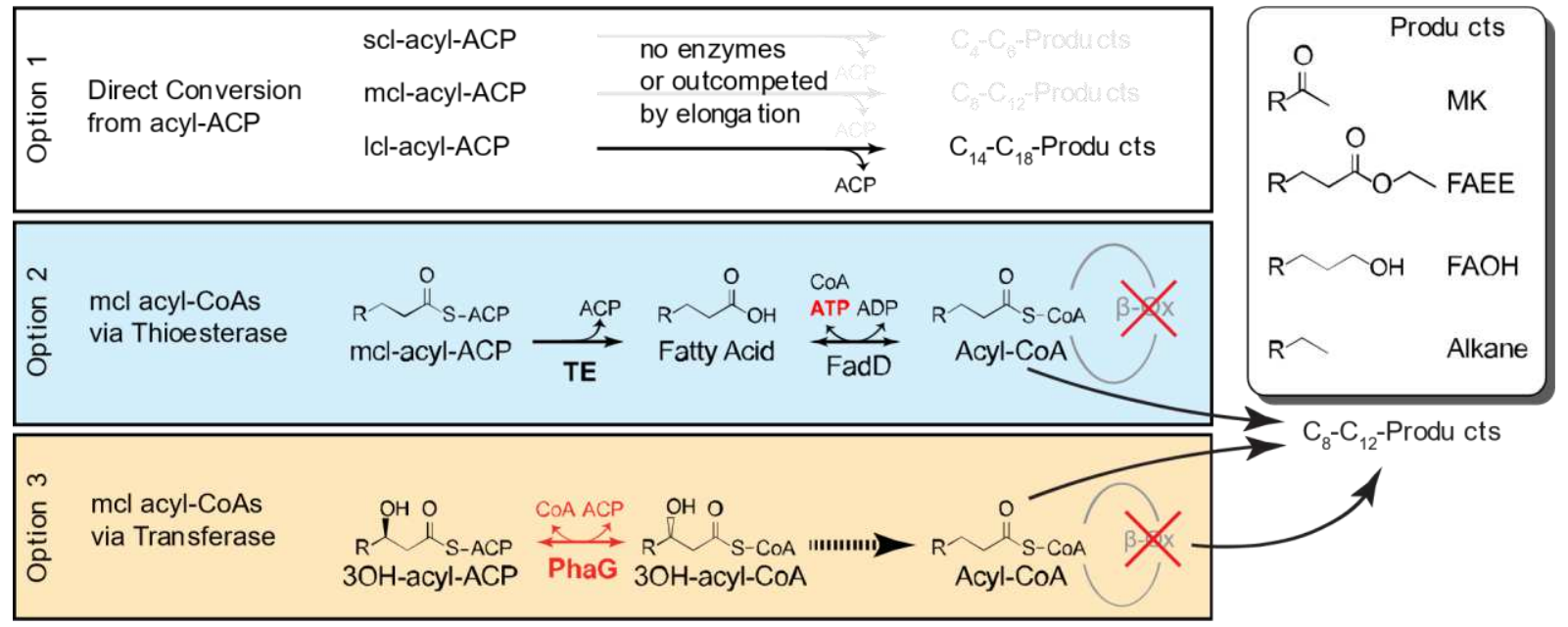

Figure 1 Metabolic pathways for producing medium-chain oleochemicals via fatty acid biosynthesis. Oleochemical products such as methyl ketones (MK), fatty acid ethyl esters (FAEE), fatty alcohols $(\mathrm{FAOH})$, and alkanes are made by enzymes that act on acyl-thioesters. Many oleochemical-producing enzymes can act on long-chain acyl-ACPs (Option 1) but fail to produce medium or short-chain products in high amounts without additional genetic modifications. The reduced activity is likely caused by lower concentrations of shorter chain length acyl-ACPs and/or reduced specific activity on these substrates, particularly when compared to enzymes in fatty acid biosynthesis that elongate the same substrates. Alternatively, if $\beta$-oxidation is blocked (often by deleting enzymes catalyzing the appropriate step in the cycle), higher concentrations of mcl-acylCoA's can be provided to saturate oleochemical-producing enzymes. This acyl-CoA pool can be created either by the combination of thioesterase (TE) and acyl-CoA synthetase (e.g. FadD) activity (Option 2) or by acyl-transferase activity (Option 3). Transferase activity (e.g. PhaG) saves one ATP per product relative to Option 2, leading to higher theoretical yields. Here, we have focused on transfer of 3-hydroxyacyl-thioesters that can be converted to desired substrates for oleochemical producing enzymes with additional enzymes (represented by a bold dashed arrow). Detailed pathways for Options 2 and 3 are illustrated in Supplementary Figure S1.

\subsection{Genetic Studies Confirm PhaG is an Acyl-ACP:CoA Transacylase}

Homologs of ${ }^{P p u} \mathrm{PhaG}$ have been used extensively as a means of enhancing production of medium-chain length (mcl) PHA in bacteria ${ }^{19,28-32}$. That said, the specific activities catalyzed by ${ }^{P p}{ }^{p} \mathrm{PhaG}$ are less well cataloged. Prior studies connected PhaG expression with the production of hydroxy fatty acids ${ }^{32,33}$ and suggested that PhaG catalyzes 3-hydroxyacyl-ACP thioesterase activity. If true, the metabolic yield advantages motivating this work would be lost. Therefore, we compared the metabolic product profiles (looking for production of methyl ketones or hydroxy 
fatty acids) of specifically engineered strains of E. coli (MG1655 $\Delta f a d A, \Delta f a d I, \Delta f a d D, \Delta f a d R$, pTRC99a- ${ }^{P p u} p h a G-{ }^{E c} f a d M$ ) to determine if heterologously expressed PhaG has thioesterase and/or transacylase activity. Strains were designed to create a 3-hydroxy fatty acid product sink to indicate potential PhaG thioesterase activity and a methyl ketone product sink for PhaG transacylase activity (Figure 2A). Deletion of $f a d D$ removes acyl-CoA synthetase activity and prevents reactivation of free fatty acids generated by either FadM or PhaG. Deletion of fadA and fadI removes known thiolase activities from $E$. coli and blocks $\beta$-oxidation from catabolizing any acylCoAs produced in vivo. Deletion of $f a d R$ removes repression of $f a d B$ expression and thereby upregulates a bi-functional enoyl-CoA hydratase/3-hydroxyacyl-CoA dehydrogenase responsible for converting hydroxy-acyl-CoA thioesters to 3-ketoacyl-CoA thioesters. FadM ${ }^{4,34}$ is overexpressed to provide 3-ketoacyl-CoA thioesterase activity, resulting in conversion of any 3hydroxyacyl-CoAs generated by PhaG to the corresponding methyl ketones. Cultures of E. coli RADI strain harboring pTRC99a- ${ }^{P p u} p h a G_{-}{ }^{E c} f a d M$ were grown in Clomburg media at $30^{\circ} \mathrm{C}$ for 48 h. Culture samples were extracted and derivatized for GC/FID and GC/MS analysis. The samples contained a total of $170 \mathrm{mg} / \mathrm{L} \mathrm{C} \mathrm{C}_{7}-\mathrm{C}_{13}$ methyl ketones but no detectable 3-hydroxy methyl esters (Figure 2B) indicating that ${ }^{P p u} \mathrm{PhaG}$ functions primarily as a 3-hydroxyacyl ACP:CoA transacylase. Strains expressing FadD (E. coli RAI strain pTRC99a $\left.{ }^{P p u} p h a G_{-}{ }^{E c} f a d M\right)$ produced equivalent amounts of methyl ketones, indicating that the carbon flux for methyl ketone synthesis is primarily from the activity of PhaG rather than activity of free fatty acid reactivation by FadD. Strains lacking PhaG overexpression (E. coli RADI strain pTRC99a- $\left.{ }^{E c} f a d M\right)$ produced small amounts $(<1 \mathrm{mg} / \mathrm{L}$ ) of methyl ketones (Figure 2B) that have been previously observed in strains expressing FadM in E. coli ${ }^{4,34}$. Strains lacking ${ }^{E c}$ FadM overexpression (E. coli RADI strain pTRC99a- ${ }^{P p u} p h a G$ ) contained a total of $55 \mathrm{mg} / \mathrm{L} \mathrm{C}_{8}-\mathrm{C}_{12}$ 3-hydroxy methyl esters (Figure 2C). 
Together, this data suggests that 3-hydroxy fatty acid production observed in past studies likely comes from thioesterase activities encoded by native enzymes (e.g. YciA, FadM, TesB can potentially catalyzes $(R)$-3-hydroxyacyl-CoA to 3-hydroxy fatty acids) that are outcompeted by the methyl ketone synthesis pathway we introduced.

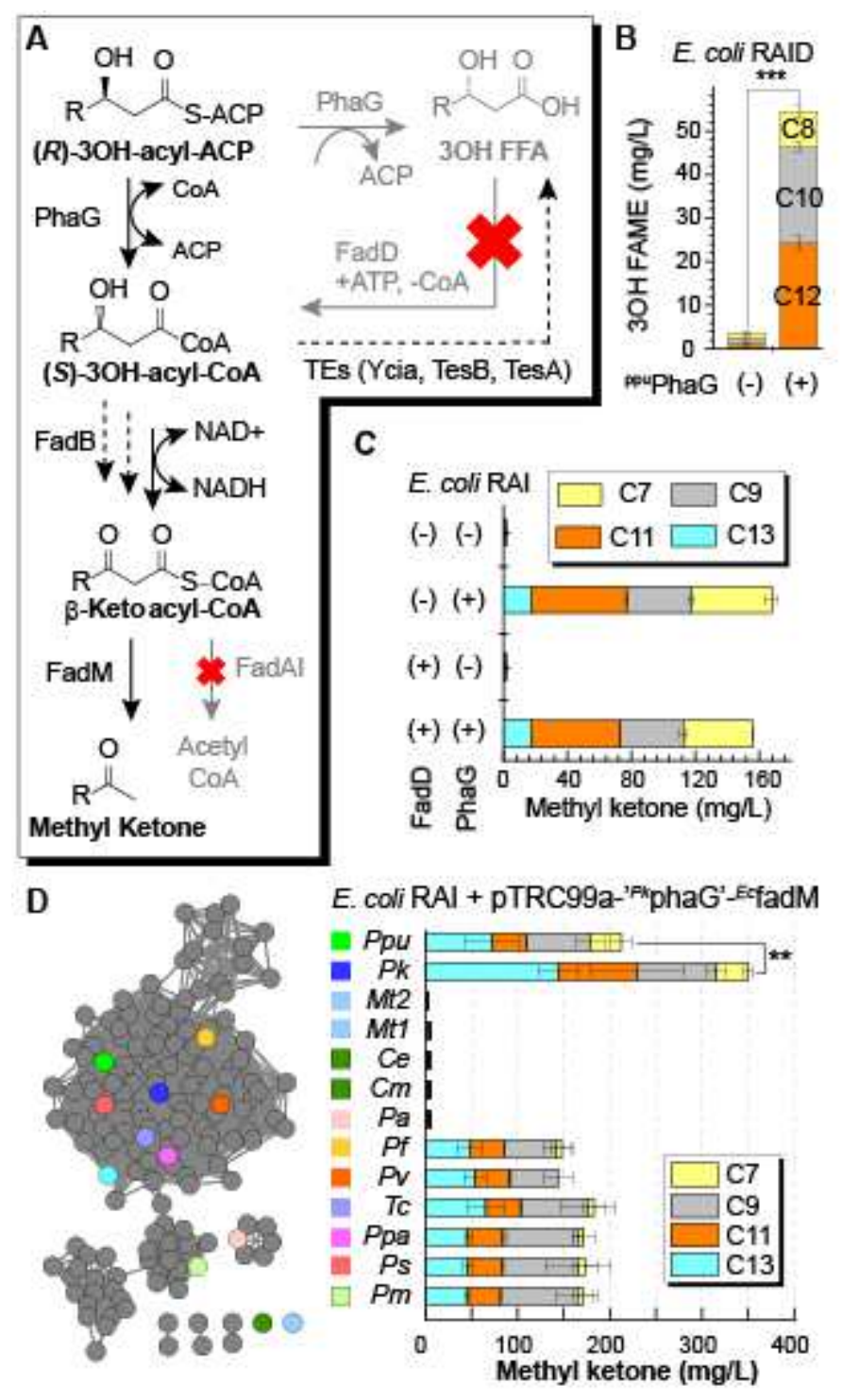

Figure 2 (A) Designed pathways for testing PhaG activities for methyl ketone or 3-hydroxy fatty acid synthesis. Two potential activities of PhaG is proposed, a thioesterase that converts $(R)-3$ hydroxyacyl-ACP to 3-hydroxy fatty acids or an acyl-ACP:CoA transacylase that transfers $(R)-3-$ hydroxyacyl moiety from a ACP to CoA (B) - (C) Methyl ketone or 3-hydroxy fatty acid produced by E. coli strain RAI harboring pTRC99a- ${ }^{P p u} p h a G-{ }^{E c} f a d M$, pTRC99a- ${ }^{E c} f a d M$ or pTRC99a${ }^{P p u} p h a G$. Analysis of methyl ketone production with or without chromosomal ${ }^{E c} \mathrm{FadD}$ deletion 
using E. coli RAI strain harboring pTRC99a $-{ }^{P p} p h a G-{ }^{E c} f a d M$. (n=3). 3-hydroxy fatty acid produced by $E$. coli strain RADI harboring pTRC99a- ${ }^{P p u} p h a G(\mathrm{n}=3)$. (D) Sequencing similarity map of bioprospecting ${ }^{P p u} \mathrm{PhaG}$. Selected homologs are colored. Quantitative pairwise percent amino acid identity multiple alignment of each enzyme homolog can be found in Figure S2 (E) Mean methyl ketone titers for constructs of PhaG homologs using E. coli RAI harbor pTRC99a'Pk phaG' ${ }^{-E c} f a d M(\mathrm{n}=3)$. Cultures were cultivated in the Clomburg medium containing $20 \mathrm{~g} / \mathrm{L}$ glycerol at $30^{\circ} \mathrm{C}$ and shaking at 250 r.p.m. All error bars represent standard error. ** represents $\mathrm{P}$ value $<0.01$ analyzed based on student $t$-test assuming unequal variances.

\subsection{Bioprospecting Identifies Active PhaG Variants}

Next, we sought to identify higher activity variants through bioprospecting. We conducted a homology search based on the Pseudomonas putida KT2440 PhaG ( $\left.{ }^{P p u} \mathrm{PhaG}\right)$ sequence using the Basic Local Alignment Search Tool (BLAST) ${ }^{35}$ to identify candidate protein sequences. BLAST hits were sorted using the Enzyme Similarity Tool ${ }^{36}$. Among the homologous sequences that have similarity greater than $45 \%$, we found $>95 \%$ of sequences belong to Pseudomonas species, indicating that the transacylase PhaG enzyme is quite unique in Pseudomonads even compared to other bacteria that have reported PHA synthesis ${ }^{37}$. We selected thirteen PhaG homologs which had a protein sequence similarity range of $24-88 \%$ based on a pairwise comparison of each transacylase, shown in Figure 2D and Supplementary Figure S2. We were particularly interested in homologs from Mycobacteria and Corynebacteria species because of their potential to interface with substrates linked to the ACP domain of type I fatty acid synthase (FAS) found in these species $^{38}$. The activity of PhaG homologs was assayed in vivo by monitoring methyl ketone production using E. coli RADI harboring a pTRC99a- ${ }^{P k} p h a G{ }^{2}{ }^{E c} f a d M$ plasmid. Most PhaG variants generated similar methyl ketone titers to ${ }^{P p u} \mathrm{PhaG}$ albeit with reduced levels of 2heptanone. The PhaG variants from Mycobacteria and Corynebacteria failed to produce methyl ketones. We found that the Pseudomonas koreensis ${ }^{P k} \mathrm{PhaG}$ showed the highest production at 350 $\mathrm{mg} / \mathrm{L}$ total methyl ketone, 1.6 times higher than the ${ }^{P p u} \mathrm{PhaG}$, shown in Figure 2D. The methyl 
ketone profile included $34 \mathrm{mg} / \mathrm{L}$ 2-heptanone, $85 \mathrm{mg} / \mathrm{L}$ 2-nonanone, $85 \mathrm{mg} / \mathrm{L}$ 2-undecanone and $145 \mathrm{mg} / \mathrm{L}$ 2-tridecanone indicating a broad activity against medium-chain 3-hydroxyacyl-ACPs.

\subsection{Metabolic Engineering to Enhance Methyl Ketone Production}

A central tenet of metabolic engineering states that enzyme activity must be balanced across a metabolic pathway to minimize unwanted accumulation of intermediates and maximize pathway flux. In order to assess the relative activity of PhaG to pathway flux, we varied cooverexpression of ${ }^{P p u} \mathrm{PhaG}$, the more-active ${ }^{P k} \mathrm{PhaG}$, and enzymes that convert 3-hydroxyacylCoAs to 3-ketoacyl-CoAs. In particular, we were concerned about the relative activity of ketoreductases on the two 3-hydroxyacyl-CoA stereoisomers. PhaG generates (R)-3-hydroxyacylCoA for direct polymerization into PHA. In contrast, $\beta$-oxidation passes through (S)-3hydroxyacyl-CoAs. FadB, the dual-function enoyl-CoA hydratase and dehydrogenase, can isomerize $(R)$-3-hydroxy-acyl-CoA via the corresponding enoyl-acyl-CoA, likely at a reduced rate relative to its preferred substrate isomer ${ }^{39-42}$. PHA producing bacteria solve this problem by expressing an alternative enoyl-CoA hydratase (PhaJ) that can generate the preferred $R$-isomer (Figure 3A) from $\beta$-oxidation intermediates. We selected ${ }^{E c} \mathrm{FadB}$ and ${ }^{E c} \mathrm{FadJ}$, an anaerobically expressed FadB homolog, and four previously studied PhaJ variants from P. aeruginosa ${ }^{26}$. These enzymes were important optimization points because deletion of $f a d B$ and $f a d J$ eliminated PhaGdependent methyl ketone production (Figure 3B).

A combination of ${ }^{E c} \mathrm{FadB},{ }^{E c} \mathrm{FadB} /{ }^{E c} \mathrm{FadJ}$ and four ${ }^{P p u} \mathrm{PhaJ}$ homologs were cloned into an operon linked to a $\mathrm{P}_{\mathrm{TRC}}$ promoter on a pACYC vector. Each of these vectors was co-expressed with pTRC99a- ${ }^{P p u} p h a G-{ }^{E c} f a d M$ or pTRC99a- ${ }^{P k} p h a G-{ }^{E c} f a d M$ in E. coli RAI. Cultures of each strain were grown at $30^{\circ}$ for 48 hours. Methyl ketones were extracted from culture samples and 
quantified by GC/FID (Figure 3). Strains expressing ${ }^{P p u} \mathrm{PhaG}$ all produced $\sim 160 \mathrm{mg} / \mathrm{L}$ of methyl ketones with similar distributions to prior experiments. In contrast, when ${ }^{P k} \mathrm{PhaG}$ was expressed, methyl ketone titers increased 2-3 fold relative to the corresponding ${ }^{P p u} \mathrm{PhaG}$ strains. In this series, co-expression of PhaJ1 and PhaJ3 had the biggest impact on methyl ketone titer, surpassing 0.5 $\mathrm{g} / \mathrm{L}$ in cultures of the best strains. These experiments indicate that 3-hydroxyacyl-CoA isomerization can be a limiting step when PhaG transacylase activity is increased. Further, these experiments suggested that titers could be improved further with more active PhaG enzymes.

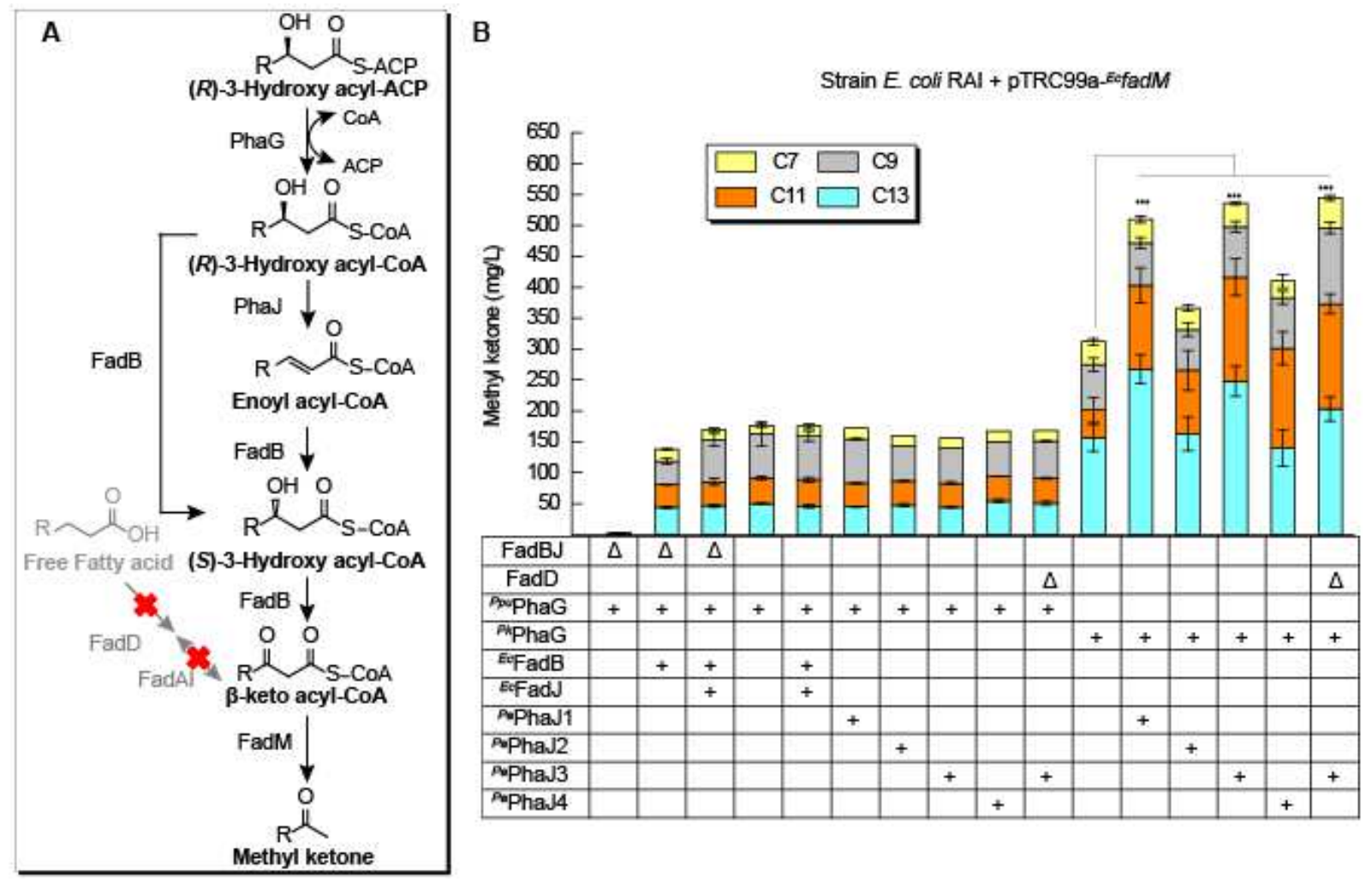

Figure 3 (A) Scheme of in vivo characterization of enzymes catalyzing reactions that bridge between $(R)$-3-hydroxyacyl-CoAs and acyl-CoA intermediates in $\beta$-oxidation pathway. (B) Evaluation of methyl ketone productions based on stepwise genetic modifications from basal strains RAI and RABIJ $(\mathrm{n}=3)$. All error bars represent standard error. ${ }^{* * *}$ represents $\mathrm{P}$ value $<$ 0.001 analyzed based on student $t$-test assuming unequal variances. 


\subsection{Random mutagenesis PhaG and selection for lipoic acid requirement of $E$. coli}

Our initial experiments indicated that PhaG has the capability of controlling the synthesis of medium-chain oleochemicals analogous to acyl-ACP thioesterases. However, unlike thioesterases, our bioprospecting study did not identify PhaG variants with dramatically different selectivities to medium-chain species. Therefore, we initiated a protein engineering study to seek variants with altered specificity and enhanced activity. We constructed an error-prone PCR library of ${ }^{P k} p h a G$ ORFs and screened them for the ability to complement a lipoic acid auxotrophy. The lipoic acid auxotrophy was recently used to isolate $C_{8}$-specific thioesterases with enhanced $V_{\max }{ }^{14}$. E. coli CM23 strain ${ }^{11}$, (MG1655 $4 a r a B A D ~ \triangle f a d A B, \triangle f a d I J, \triangle f a d D, \Delta f a d R, \Delta f a d E, \Delta l d h A$, $\triangle a c k A p t a, \triangle a d h E, \triangle p o x B, \triangle f r d A B C D, \triangle y d i O, \triangle a t o C)$, which lacks all $\beta$-oxidation genes and many fermentation pathways, was chosen as the base strain as it lacks all alternative routes to lipoic acid. We constructed an E. coli CM23- $\Delta$ lipB strain to implement the lipoic acid auxotrophy. This strain requires supplementation of lipoic acid or octanoic acid in the media to enable the formation of an active pyruvate dehydrogenase and aerobic growth on glucose. To link the octanoic acid selection to PhaG activity, we added heterologous enzymes to convert the 3-hydroxyoctanoyl-CoA to octanoic acid (Figure 4A). In order to allow CM23- $\triangle$ lipB to synthesize free fatty acids, we coexpressed genes encoding ${ }^{P k} \mathrm{PhaG},{ }^{P a} \mathrm{PhaJ} 3$, a Treponema denticola reductase ${ }^{T d}{ }^{\mathrm{TER}}{ }^{11}$ and a Mycobacterium sp. acyl-CoA thioesterase ${ }^{M a}$ TesB A197D (referred as to $\left.{ }^{M a} \mathrm{TesB}^{*}\right)^{43}$ (Figure 4A). Purified Mycobacterium avium ${ }^{M a}{ }^{T}$ esB* has been shown to hydrolyze octanoyl-CoA and generate octanoic acid in vitro ${ }^{43}$. We neglected the octanoic acid synthesis activities by endogenous $E$. coli acyl-CoA thioesterase since ${ }^{E_{c}}$ TesB generally has activities toward longer chain acyl-CoA $(>\mathrm{C} 10)^{44}$ and ${ }^{E c}$ YciA has activities toward shorter chain acyl-CoA $\left(<\mathrm{C}_{8}\right){ }^{45,46}$. To test the basal 
octanoic acid production level, we found E. coli CM23- $\operatorname{lipB}$ harboring pTRC99a $-{ }^{P k} p h a G-{ }^{T d} T E R$ + pACYC- ${ }^{P a}$ phaJ3 + pBTRCK $-{ }^{M a}$ tes $B^{*}$ plasmids produced $\sim 20 \mathrm{mg} / \mathrm{L}$ octanoic acid after 48 hours, while the corresponding strain without ${ }^{P k} \mathrm{PhaG}$ produced $<1 \mathrm{mg} / \mathrm{L}$ octanoic acid (data not shown). In order to reduce the baseline octanoic acid titer, we subcloned ${ }^{P k} p h a G$ onto a low copy vector $(\mathrm{pBTRCK}){ }^{11}$ and ${ }^{M a}$ tes $B^{*}$ onto a high copy number vector pTRC99a. The latter was performed to ensure that octanoic acid production would be limited solely by PhaG activity. After tuning the copy number of ${ }^{P k} p h a G$ and ${ }^{M a}$ tes $B^{*}$, E. coli CM23-AlipB strain harboring pTRC99a- ${ }^{M a}$ tes $B^{*}$ ${ }^{T d}$ TER + pACYC- ${ }^{P a}{ }^{p h a J 3}+$ pBTRCK- ${ }^{P k}$ phaG' plasmids produced $\sim 7 \mathrm{mg} / \mathrm{L}$ octanoic acid after 48 hours. Selections of the error-prone PhaG library were performed using E. coli CM23- $\Delta$ lipB strain harboring pTRC99a- ${ }^{M a} t e s B^{*}{ }^{T d} T E R+$ pACYC- ${ }^{P a} p h a J 3+$ pBTRCK- ${ }^{P k} p h a G$.

In the first-round of mutagenesis, hundreds of colonies appeared on MOPS-glucose minimal media plates containing $20 \mu \mathrm{M}$ IPTG three days after plating. On day 4 , we picked $\sim 180$ of the largest colonies and quantified the octanoic acid titer from individual liquid cultures grown in Clomburg liquid media containing $20 \mathrm{~g} / \mathrm{L}$ glycerol. We found that 17 strains expressing ${ }^{P k} \mathrm{PhaG}$ variants increased octanoic acid titer 3.3- to 16.3-fold over the parent ${ }^{P k} \mathrm{PhaG}$ (Figure S3). All 17 ${ }^{P k} \mathrm{PhaG}$ mutant strains increased total fatty acid production ranging 1.8 - to 8.3 -fold with modest changes in the overall distribution of chain-lengths.

To investigate why titers were increased in these strains, we examined PhaG abundance using a translational coupling circuit linking RFP to $\mathrm{PhaG}^{47}$. We compared expression of five PhaG variants (WT, LM1, LM7, LM9 and LM14) by proxy using RFP fluorescence generated by the translationally coupling cassette. When YFP was cloned in place of PhaG, RFP fluorescence was proportional to YFP fluorescence over a range of IPTG (inducer) concentrations (Supplementary Figure S4). PhaG variants LM9, LM14, and WT demonstrated equivalent 
fluorescence values across a range of IPTG concentrations. PhaG variant LM7 generated slightly more RFP fluorescence, indicating it may be present at higher concentrations. Conversely, PhaG variant LM1 produced $\sim 60 \%$ of the RFP fluorescence of the WT. Decreased expression may be due to the occurrence of a rare codon CGG at point mutation Q45R. CGG codons have been shown in other studies to be a rate-limiting factor for translational efficiency due to its low abundance tRNA supply in E. coli ${ }^{48,49}$. We estimated specific PhaG activity by normalizing the sum of observed $\mathrm{C}_{8}-\mathrm{C}_{14} \mathrm{FAME}$ titer to RFP fluorescence (proxy for protein abundance). These estimates indicate the LM1 and LM14 variants are 7.4- and 8.8-fold more active than wild-type PhaG (Supplementary Figure S4).

From the sequences of the 17 improved ${ }^{P k} \mathrm{PhaG}$ variants, we identified 28 point mutations. To investigate the importance of each on ${ }^{P k} \mathrm{PhaG}$ activity, we created the full library of single point mutations. We coexpressed each ${ }^{P k} \mathrm{PhaG}$ variant from the high copy number pTRC99a- ${ }^{P k} p h a G^{\prime}-$ ${ }^{T d} T E R$ vector with $\mathrm{pACYC}-{ }^{P a}$ phaJ3 $+\mathrm{pBTRCK}^{\mathrm{Ma}}{ }^{\text {tes }} B^{*}$ in $E$. coli CM23. Each strain was cultured for $48 \mathrm{hrs}$ at $30^{\circ} \mathrm{C}$ and samples were harvested for fatty acid quantification. We found that variants containing 6 of the 28 single point mutations (e.g. Q45R, R66H, H76Y, Y138F, G142V, Q277X) increased octanoic acid titer more than 2-fold over strains expressing the parent ${ }^{P k} \mathrm{PhaG}$ (Supplementary Figure S5). Among these six single point mutations, three individual ${ }^{P k} \mathrm{PhaG}$ mutants Q45R, G142V and Y138F strains had 1.9-, 1.8- and 1.5-fold higher total fatty acid titers than strains expressing wild-type ${ }^{P k} \mathrm{PhaG}$.

In order to evaluate the combinatorial effects of the three most beneficial single point mutations, we constructed all four possible combinations of Q45R, G142V and Y138F mutations in a vector pTRC99a- ${ }^{P k} p h a G{ }^{\prime}{ }^{T d} T E R$. These combinatorial mutants were co-expressed with pACYC- ${ }^{P a}$ phaJ3 + pBTRCK $^{M a}{ }^{\text {tes }} B^{*}$ in E. coli $\mathrm{CM} 23$. Each strain was cultured for $48 \mathrm{hrs}$ at $30^{\circ} \mathrm{C}$ 
and samples were harvested for fatty acid quantification. Overall, the best variant, ${ }^{P k} \mathrm{PhaG}$ Q45R G142V, strain produced the highest amount of $\mathrm{C}_{8}-\mathrm{C}_{14}$ free fatty acid at $1.1 \mathrm{~g} / \mathrm{L}$, a 4.0 -fold increase of total $\mathrm{C}_{8}-\mathrm{C}_{14}$ free fatty acid compared to that of the wild-type ${ }^{P k} \mathrm{PhaG}$ (Figure 4B). The fatty acid pool contained $41 \%$ tetradecanoic acid, 22\% dodecanoic acid, 11\% decanoic acid, and 26\% octanoic acid.

\subsection{Validating enzymes converting $(R)-3$-hydroxyacyl-CoA intermediates}

In order to re-evaluate the effect of FadB and PhaJ activities on overall rate of the PhaG pathway with the more active ${ }^{P k} \mathrm{PhaG}^{*}$ (referred as to ${ }^{P k} \mathrm{PhaG}$ Q45R G142V) variant, we cloned six FadB homologs and four PhaJ homologs in a pACYC vector, co-expressed each with pTRC99a- ${ }^{T d}$ TER- ${ }^{P k} p h a G^{*}$ and $\mathrm{pBTRCK}-{ }^{M a}$ tes $B^{*}$ in strain CM23, and cultured them for fatty acid production. The strain expressing ${ }^{P a} \mathrm{PhaJ} 3$ and ${ }^{P a} \mathrm{PhaJ} 1$ produced the highest fatty acid titers, suggesting these enzymes have the highest activity for converting $(R)$-3-hydroxyacyl-CoA substrate to enoyl-acyl-CoA (Figure 4C). We found that all FadB variants produced substantially lower titers compared to the analogous strains expressing ${ }^{P a} \mathrm{PhaJ} 3$. This suggests that FadB has lower activity on recognizing $(R)$-3-hydroxyacyl-CoAs than ${ }^{P a} \mathrm{PhaJ} 3$. This is not surprising since in Pseudomonas, FadB might evolve to lower its isomerization activity to avoid crosstalk between PHA biosynthesis and beta-oxidation. 


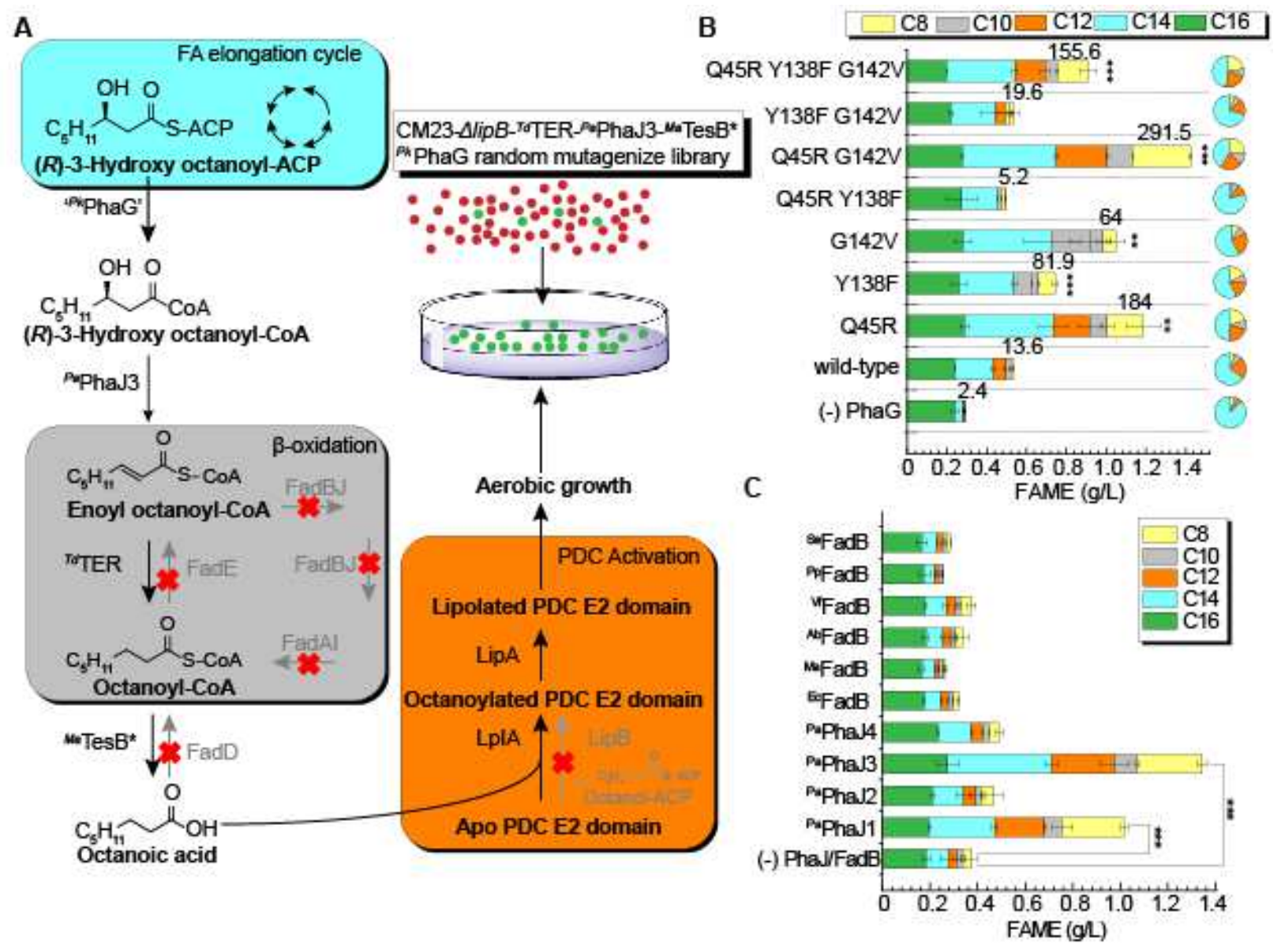

Figure 4 (A) Scheme of octanoic acid/lipoic acid selection strategies based on E. coli CM23- $\triangle$ lipB strain. ${ }^{E c} \mathrm{FadD}$ deletion blocks reactivation of fatty acids, ${ }^{E c} \mathrm{FadE}$ deletion blocks hydrogenation enoyl octanoyl-CoA to octanoyl-CoA, ${ }^{E c}$ FadBJ deletion blocks hydratation and dehydrogenation of enoyl octanoyl-CoA to $\beta$-ketooctanoyl-CoA by ${ }^{E c} \mathrm{FadBJ},{ }^{E c} \mathrm{FadAI}$ deletion blocks condensation of octanoyl-CoA and acetyl-CoA to $\beta$-ketodecanoyl-CoA, LipB deletion blocks activation of apo PDC E2 domain to octanoylated PDC E2 domain. The PhaG enzyme was designed to pull carbon flux from 3-hydroxyoctanoyl-ACP to $(R)$-3-hydroxyoctanoyl-CoA. (R)-3-hydroxyoctanoyl-CoA undergoes a dehydration and hydrogenation process by ${ }^{P a} \mathrm{PhaJ} 3$ and ${ }^{T d}$ TER to generate octanoylCoA. Octanoyl-CoA is cleaved by ${ }^{M a} \mathrm{TesB}^{*}$ to release ACP and produce octanoic acid. The produced octanoic acid is the octanoyl donor for the octanoylation process of Apo PDC E2 domain to octanoylated PDC E2 domain. When octanoic acid is produced by LipB deletion CM23 strain by the designed PhaG pathway, cells were capable of grown on minimal MOPS agarose plates. ${ }^{M a}$ TesB* and ${ }^{T d}$ TER were cloned in a high copy vector $\mathrm{pTRC}$ T9a, ${ }^{P a} \mathrm{PhaJ} 3$ was cloned in a medium copy vector pACYC, ${ }^{P k} \mathrm{PhaG}$ was cloned in a low copy vector pBTRCK. (B) FAME profiles of 17 hits identified in the genetic selection strategy of lipoic acid requirement of E. coli using a randomly mutagenized. The strain CM23 harboring pBTRCK- ${ }^{P k}{ }^{p h a G}{ }^{\prime}+$ pACYC- ${ }^{P a}$ phaJ3 + pTRC99a- ${ }^{T d}$ TER- ${ }^{M a}$ tes $B^{*}$ were cultured in test tubes containing $5 \mathrm{~mL}$ Clomburg $20 \mathrm{~g} / \mathrm{L}$ glycerol and $1 \mathrm{mM}$ IPTG at $30^{\circ} \mathrm{C}$ for $48 \mathrm{hrs}$. (C) Combinatorial effects of single point mutations on PhaG activities in vivo for fatty acid production $(\mathrm{n}=3)$. All error bars represent standard error. ** means 
$\mathrm{P}$ value $<0.01$ and $* * *$ means $\mathrm{P}$ value $<0.001$ analyzed based on student $\mathrm{t}$-test assuming unequal variances.

\subsection{Validate methyl ketone and fatty alcohol production}

With an enhanced 3-hydroxyacyl-ACP:CoA transacylase ${ }^{P k} \mathrm{PhaG}$ mutant and a more active (R)-3-hydroxyacyl-CoA dehydratase ${ }^{P a} \mathrm{PhaJ} 3$ selected, we wanted to benchmark the PhaG pathway for methyl ketone and fatty alcohol production. In order to produce methyl ketones, we cultured E. coli RADI harboring pTRC99a- ${ }^{P k} p h a G^{*}{ }^{P s} f a d M+$ pACYC- ${ }^{P a} p h a J 3$ plasmids. A $\beta$ ketoacyl-CoA thioesterase ${ }^{P_{s}} \mathrm{FadM}$ from Providencia sneebia was chosen for its high activity toward medium-chain $\beta$-ketoacyl-CoA substrates. The strain RADI harboring pTRC99a- ${ }^{P k} p h a G^{*}{ }_{-}$ ${ }^{P} f a d M+$ pACYC- ${ }^{P a}$ phaJ3 was incubated for 72 hours at $30{ }^{\circ} \mathrm{C}$ in shake flasks containing rich glycerol media. The cultures produced $1.5 \mathrm{~g} / \mathrm{L}$ total methyl ketone from $20 \mathrm{~g} / \mathrm{L}$ glycerol (Figure 5A), representing 26\% 2-heptanone, 27\% 2-nonanone, 19\% 2-undecanone, 14\% 2-tridecanone, 14\% 2-heptadecanone. To produce fatty alcohols via PhaG activity, we cultured strain E. coli RADI harboring pTRC99a- ${ }^{P k} p h a G^{*}{ }_{-}^{T d}$ TER + pACYC- ${ }^{P a} p h a J 3+$ pBTRCK- ${ }^{M a} A C R$ for 72 hrs at $30{ }^{\circ} \mathrm{C}$ in shake flasks containing rich glycerol media. The strain produced $\sim 1.1 \mathrm{~g} / \mathrm{L}$ total fatty alcohol from 20 g/L glycerol (Figure 5B), representing 6\% 1-octanol, 6\% 1-decanol, 7\% 1dodecanol, 41\% 1-tetradecanol, 41\% 1-hexadecanol. The titer and yield of fatty acids, methyl ketones and fatty alcohols from the PhaG-dependent strategies are comparable to values reported in literature using thioesterase strategies or reverse $\beta$-oxidation strategies, shown in Table 1. 

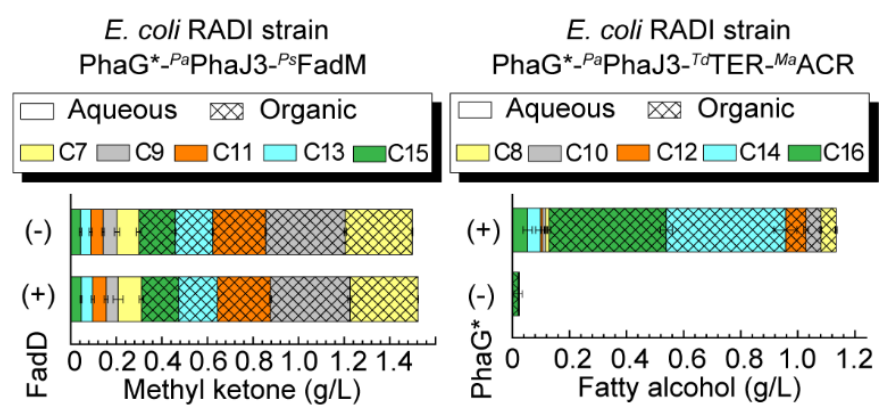

Figure 5. Benchmark of methyl ketone and fatty alcohol production in batch mode shake flask cultures. (A-B) Methyl ketone production by RADI or RAI strain harboring pTRC99a- ${ }^{P k} p h a G^{*}$ ${ }^{P} f a d M+$ pACYC- ${ }^{P a}$ phaJ3 plasmids. (C-D) Fatty alcohol production by RADI strain harboring pTRC99a- ${ }^{P k}$ phaG ${ }^{*}{ }^{P S} f a d M+$ pACYC- ${ }^{P a} p h a J 3+$ pBTRCK- ${ }^{M a} A C R$ and negative control corresponding strain without coexpressing $\mathrm{PhaG}^{*}$. All cultures were cultivated in $50 \mathrm{~mL}$ Clomburg media $20 \mathrm{~g} / \mathrm{L}$ glycerol and $1 \mathrm{mM}$ IPTG at $30^{\circ} \mathrm{C}$ for $48 \mathrm{hrs}(\mathrm{n}=3)$. All error bars represent standard error.

\subsection{Methyl ketone production in bioreactors}

In effort to demonstrate enhanced methyl ketone titers, we performed fed-batch cultivations in a stirred bioreactor by adding Clomburg media + glycerol pulses after cells reached high cell densities. We followed two previously developed strategies to reduce the loss of mediumchain methyl ketones in the off-gas by adding a $20 \%$ dodecane overlay to the culture ${ }^{4}$. After 96 hrs of cultivation, cells consumed $90.0 \mathrm{~g} / \mathrm{L}$ of glycerol, reached an $\mathrm{OD}_{600} \sim 60$, and produced 6.8 $\mathrm{g} / \mathrm{L}$ total methyl ketone titer (Figure 6A). Additionally, we used ASPEN to evaluate and design improved gas traps to capture product leaving in the off-gas (Supplementary Figure S6) ${ }^{4}$. We bubbled off-gas through a jacketed gas dryer filled with dodecane at $5^{\circ} \mathrm{C}$ and observed similar glycerol consumption, biomass formation, and methyl ketone production rates within the reactor. After $96 \mathrm{hrs}$ of induced cultivation, cells reached a density of $\mathrm{OD}_{600} \sim 60$, consumed $95.2 \mathrm{~g} / \mathrm{L}$ glycerol, and produced $6.7 \mathrm{~g} / \mathrm{L}$ total methyl ketone titer (Figure 6B). At the end-point, we observed $3.4 \mathrm{~g} / \mathrm{L}$ methyl ketone contained in the condensed dodecane phase, corresponding to an additional $0.51 \mathrm{~g} / \mathrm{L}$ (culture volume) of total methyl ketone captured from the bioreactor (Figure 6C). 

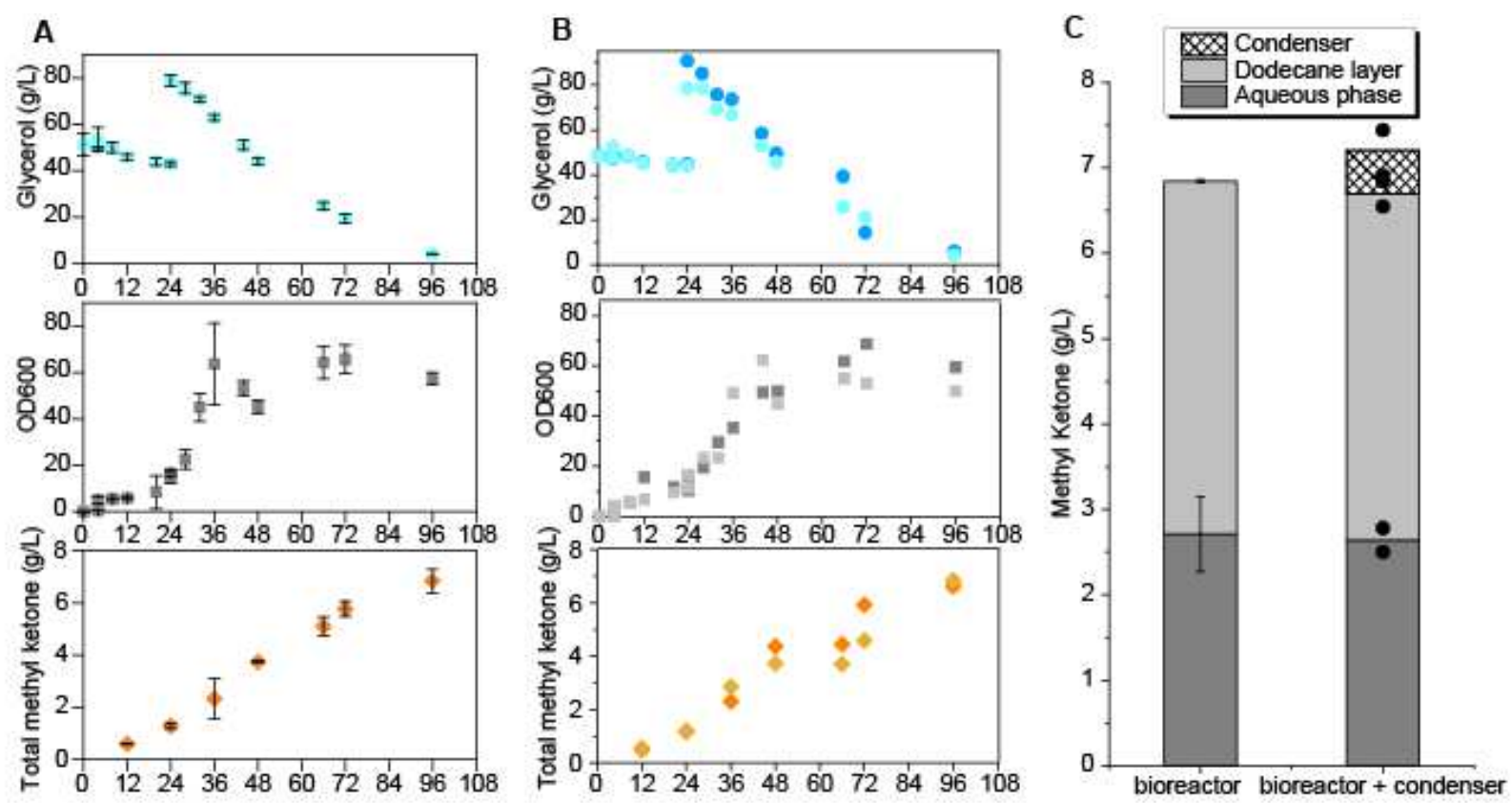

Figure 6. Time course of concentration of glycerol, $\mathrm{OD}_{600}$ and total methyl ketone in fed-batch fermentation from bioreactor $(\mathrm{n}=3)(\mathbf{A})$ and bioreactor coupling a condenser $(\mathrm{n}=2)(\mathbf{B})$ using $E$. coli RADI strain harbors pTRC99a- ${ }^{P k} p h a G^{*}{ }_{-}{ }^{P s} f a d M$ and pACYC- ${ }^{P a} p h a J 3$ plasmids. (C) Evaluation of methyl ketone concentration from samples in dodecane layer, aqueous phase and condenser after 96 hours fermentation. All error bars represent standard error. 
Table 1 Reported titers and yield of oleochemical production using established thioesterasedependent strategies or developed PhaG-dependent strategies in this work

\begin{tabular}{|c|c|c|c|c|}
\hline Strategies & Products & $\begin{array}{l}\text { Titer } \\
\text { (g/L) }\end{array}$ & Yield & Fermentation \\
\hline \multicolumn{5}{|l|}{ Thioesterase } \\
\hline${ }^{\mathrm{Cp}} \mathrm{FatB}^{4}$ & $\begin{array}{l}\text { 2-heptanone, } \\
\text { 2-nonanone, } \\
\text { 2-undecanone }\end{array}$ & $\begin{array}{l}4.4 \mathrm{~g} / \mathrm{L} \\
3 \mathrm{~g} / \mathrm{L} \\
0.34 \mathrm{~g} / \mathrm{L}\end{array}$ & $\begin{array}{l}0.035 \mathrm{~g} / \mathrm{g} \text { consumed glycerol, } \\
0.028 \mathrm{~g} / \mathrm{g} \text { consumed glycerol, } \\
0.014 \mathrm{~g} / \mathrm{g} \text { consumed glycerol }\end{array}$ & fed-batch, bioreactor \\
\hline${ }^{\mathrm{Cp}} \mathrm{FatB}{ }^{18}$ & 1-octanol & 1.3 & $0.065 \mathrm{~g} / \mathrm{g}$ glycerol & batch, shake flask, \\
\hline${ }^{\mathrm{Cp}} \mathrm{FatB}{ }^{14}$ & octanoic acid & 1.7 & $0.085 \mathrm{~g} / \mathrm{g}$ glycerol & batch, shake flask, \\
\hline $\mathrm{BTE}^{50}$ & $\mathrm{C}_{12}-\mathrm{C}_{14} \mathrm{FAOH}$ & 1.6 & $0.13 \mathrm{~g} / \mathrm{g}$ glucose & fed-batch, bioreactor \\
\hline${ }^{\mathrm{Ec}}$ TesA ${ }^{51}$ & $\mathrm{C}_{11}-\mathrm{C}_{17} \mathrm{MK}$ & 9.8 & $0.171 \mathrm{~g} / \mathrm{g}$ glucose & fed-batch, bioreactor \\
\hline \multicolumn{5}{|l|}{ r-BOX } \\
\hline r-BOX ${ }^{6}$ & $\mathrm{C}_{6}-\mathrm{C}_{16} \mathrm{FAOH}$ & 1.8 & $0.2 \mathrm{~mol} / \mathrm{mol}$ consumed glucose & fed-batch, bioreactor \\
\hline r-BOX ${ }^{10}$ & $\mathrm{C}_{4}-\mathrm{C}_{10} \mathrm{FFA}$ & 4.7 & $0.31 \mathrm{~g} / \mathrm{g}$ consumed glucose & fed-batch, bioreactor \\
\hline \multicolumn{5}{|l|}{ PhaG } \\
\hline${ }^{P k} \mathrm{PhaG}$ & $\mathrm{C}_{8}-\mathrm{C}_{14} \mathrm{FFA}$ & 1.2 & $0.06 \mathrm{~g} / \mathrm{g}$ glycerol & batch, shake-flask \\
\hline${ }^{P k} \mathrm{PhaG}$ & $\mathrm{C}_{8}-\mathrm{C}_{16} \mathrm{FAOH}$ & 1.1 & $0.055 \mathrm{~g} / \mathrm{g}$ glycerol & batch, shake-flask \\
\hline${ }^{P k} \mathrm{PhaG}$ & $\mathrm{C}_{7}-\mathrm{C}_{15} \mathrm{MK}$ & 7.2 & $0.075 \mathrm{~g} / \mathrm{g}$ glycerol & fed-batch, bioreactor \\
\hline
\end{tabular}

\section{CONCLUSION}

This study demonstrated that PhaG catalyzed acyltransferase activity is capable of supporting high flux to three medium-chain oleochemical products, fatty acids, fatty alcohols, and methyl ketones. The PhaG-dependent strategy has the potential to achieve higher theoretical yields compared to the well-established thioesterase route for methyl ketone and fatty alcohol production. Here, we first validated PhaG as a 3-hydroxyacyl-ACP:CoA transacylase. We then validated methyl ketone production is driven by the activity of PhaG with a designed PhaG dependent 
pathway rather than reactivation of free fatty acids by FadD. Through bioprospecting, we identified a PhaG from Pseudomonas koreensis that demonstrated higher activity when producing methyl ketones. After expressing a random mutagenesis library of ${ }^{P k} \mathrm{PhaG}$ in a lipoic acid selecting strain of E. coli, we isolated $17 \mathrm{PhaG}$ mutants that produced octanoic acid ranging from 3.3- to 16.3-fold greater than that of wild-type PhaG. Through evaluating the effect of single point mutations and their combinations, we identified a double mutant, ${ }^{P h}$ PhaG Q45R G142V, that showed 4.0-fold higher activity towards conferring total fatty acid production than the ${ }^{P k} \mathrm{PhaG}$. Finally, based on our optimization of the PhaG-dependent pathway enzymes, we demonstrated production of 1.1 $\mathrm{g} / \mathrm{L} \mathrm{C}{ }_{8}-\mathrm{C}_{14}$ free fatty acids, $1.5 \mathrm{~g} / \mathrm{L} \mathrm{C}_{7}-\mathrm{C}_{15}$ methyl ketones, and $1.1 \mathrm{~g} / \mathrm{L} \mathrm{C}_{8}-\mathrm{C}_{16}$ fatty alcohols. In fed-batch fermentation, we benchmarked total methyl ketone titers, $7.2 \mathrm{~g} / \mathrm{L}$. In total, we have demonstrated that a PhaG dependent pathway is a viable alternative option for production of oleochemicals. 


\section{CRediT AUTHORSHIP CONTRIBUTION STATEMENT}

Qiang Yan: Conceptualization, Investigation, Data curation, Writing - original draft, Writing review \& editing. Michael A. Jindra: Investigation, Writing - review \& editing, William T. Cordell: Investigation, Writing - review \& editing. Dylan Cortney: Investigation, Writing - review \& editing. Xuanqi Chen: Investigation, Writing - review \& editing. Brian F. Pfleger: Conceptualization, Writing - original draft, Writing - review \& editing, Supervision, Funding acquisition.

\section{DECLARATION OF COMPETING INTEREST}

No conflict of interest was declared for this study.

\section{ACKNOWLEDGEMENT}

This work was funded by the DOE Center for Advanced Bioenergy and Bioproducts Innovation (U.S. Department of Energy, Office of Science, Office of Biological and Environmental Research under Award Number DE-SC0018420) and the National Science Foundation (CBET-1703504). Any opinions, findings, and conclusions or recommendations expressed in this publication are those of the author(s) and do not necessarily reflect the views of the U.S. Department of Energy or the National Science Foundation. 


\section{REFERENCES}

1. Yan, Q. \& Pfleger, B. F. Revisiting metabolic engineering strategies for microbial synthesis of oleochemicals. Metab. Eng. 58, 35-46 (2020).

2. Sarria, S., Kruyer, N. S. \& Peralta-Yahya, P. Microbial synthesis of medium-chain chemicals from renewables. Nat. Biotechnol. 35, 1158-1166 (2017).

3. Vaughn, S. F. \& Holser, R. A. Evaluation of biodiesels from several oilseed sources as environmental friendly contact herbicides. Ind. Crops Prod. 26, 63-68 (2007).

4. Yan, Q. et al. Metabolic engineering of $\beta$-oxidation to leverage thioesterases for production of 2-heptanone, 2-nonanone and 2-undecanone. Metab. Eng. 61, 335-343 (2020).

5. Longo, M. A. \& Sanromán, M. A. Production of food aroma compounds: Microbial and enzymatic methodologies. Food Technol Biotechnol 44(3), 335-353 (2006).

6. Dellomonaco, C., Clomburg, J. M., Miller, E. N. \& Gonzalez, R. Engineered reversal of the $\beta$-oxidation cycle for the synthesis of fuels and chemicals. Nature 476, 355-359 (2011).

7. Lennen, R. M. \& Pfleger, B. F. Engineering Escherichia coli to synthesize free fatty acids. Trends Biotechnol. 30, 659-667 (2012).

8. Wu, J. et al. Construction of artificial micro-aerobic metabolism for energy- and carbonefficient synthesis of medium chain fatty acids in Escherichia coli. Metab. Eng. 53, 1-13 (2019).

9. Wu, J., Zhang, X., Xia, X. \& Dong, M. A systematic optimization of medium chain fatty acid biosynthesis via the reverse beta-oxidation cycle in Escherichia coli. Metab. Eng. 41, 115124 (2017).

10. $\mathrm{Wu}, \mathrm{J}$. et al. Improving metabolic efficiency of the reverse beta-oxidation cycle by balancing redox cofactor requirement. Metab. Eng. 44, 313-324 (2017).

11. Mehrer, C. R., Incha, M. R., Politz, M. C. \& Pfleger, B. F. Anaerobic production of medium-chain fatty alcohols via a $\beta$-reduction pathway. Metab. Eng. 48, 63-71 (2018).

12. Benning, C. Mechanisms of lipid transport involved in organelle biogenesis in plant cells. Annu. Rev. Cell Dev. Biol. 25, 71-91 (2009).

13. Deng, X. et al. Structure-guided reshaping of the acyl binding pocket of 'TesA thioesterase enhances octanoic acid production in E. coli. Metab. Eng. 61, 24-32 (2020).

14. Hernández Lozada, N. J. et al. Highly active C8 -acyl-ACP thioesterase variant isolated by a synthetic selection strategy. ACS Synth. Biol. 7, 2205-2215 (2018).

15. Grisewood, M. J. et al. Computational redesign of acyl-ACP thioesterase with improved selectivity toward medium-chain-length fatty acids. ACS Catal. 7, 3837-3849 (2017).

16. Feng, Y. et al. Tuning of acyl-ACP thioesterase activity directed for tailored fatty acid synthesis. Appl. Microbiol. Biotechnol. 102, 3173-3182 (2018).

17. Voelker, T. A. \& Davies, H. M. Alteration of the specificity and regulation of fatty acid synthesis of Escherichia coli by expression of a plant medium-chain acyl-acyl carrier protein thioesterase. J. Bacteriol. 176, 7320-7327 (1994).

18. Hernández Lozada, N. J., Simmons, T. R., Xu, K., Jindra, M. A. \& Pfleger, B. F. Production of 1-octanol in Escherichia coli by a high flux thioesterase route. Metab. Eng. 61, 352-359 (2020).

19. Fiedler, S., Steinbüchel, A. \& Rehm, B. H. A. PhaG-mediated synthesis of poly(3hydroxyalkanoates) consisting of medium-chain-length constituents from nonrelated varbon sources in recombinant Pseudomonas fragi. Appl. Environ. Microbiol. 66, 2117-2124 (2000).

20. Rehm, B. H. A., Krüger, N. \& Steinbüchel, A. An metabolic link between fatty acid de novo synthesis and polyhydroxyalkanoic acid synthesis. J. Biol. Chem. 273, 24044-24051 (1998). 21. Hoffmann, N. et al. Biochemical characterization of the Pseudomonas putida 3hydroxyacyl ACP:CoA transacylase, which diverts intermediates of fatty acid de novo 
biosynthesis. J. Biol. Chem. 277, 42926-42936 (2002).

22. Li, S. et al. Microbial synthesis of functional homo-, random, and block polyhydroxyalkanoates by $\beta$-oxidation deleted Pseudomonas entomophila. Biomacromolecules 15, 2310-2319 (2014).

23. Poblete-Castro, I. et al. In-silico-driven metabolic engineering of Pseudomonas putida for enhanced production of poly-hydroxyalkanoates. Metab. Eng. 15, 113-123 (2013).

24. Matsumoto, K., Matsusaki, H., Taguchi, S., Seki, M. \& Doi, Y. Cloning and characterization of the Pseudomonas sp. 61-3 phaG gene involved in polyhydroxyalkanoate biosynthesis. Biomacromolecules 2(1), 142-7 (2001).

25. Salvachúa, D. et al. Metabolic engineering of Pseudomonas putida for increased polyhydroxyalkanoate production from lignin. Microb. Biotechnol. 13, 290-298 (2020).

26. Agnew, D. E., Stevermer, A. K., Youngquist, J. T. \& Pfleger, B. F. Engineering Escherichia coli for production of C12-C14 polyhydroxyalkanoate from glucose. Metab. Eng. 14, 705-713 (2012).

27. Gibson, D. G. et al. Enzymatic assembly of DNA molecules up to several hundred kilobases. Nat. Methods 6, 343-345 (2009).

28. Zhang, X., Lin, Y., Wu, Q., Wang, Y. \& Chen, G.-Q. Synthetic biology and genomeediting tools for improving PHA metabolic engineering. Trends Biotechnol. 38, 689-700 (2020).

29. Choi, S. Y. et al. Microbial polyhydroxyalkanoates and nonnatural polyesters. Adv. Mater. 32, 1907138 (2020).

30. Tobin, K. M., O’Leary, N. D., Dobson, A. D. W. \& O’Connor, K. E. Effect of heterologous expression of phaG (R)-3-hydroxyacyl-ACP-CoA transferase on polyhydroxyalkanoate accumulation from the aromatic hydrocarbon phenylacetic acid in Pseudomonas species. FEMS Microbiol. Lett. 268, 9-15 (2007).

31. Le Meur, S., Zinn, M., Egli, T., Thöny-Meyer, L. \& Ren, Q. Production of medium-chainlength polyhydroxyalkanoates by sequential feeding of xylose and octanoic acid in engineered Pseudomonas putida KT2440. BMC Biotechnol. 12, 53 (2012).

32. Wang, Q., Tappel, R. C., Zhu, C. \& Nomura, C. T. Development of a new strategy for production of medium-chain-length polyhydroxyalkanoates by recombinant Escherichia coli via inexpensive non-fatty acid feedstocks. Appl. Environ. Microbiol. 78, 519-527 (2012).

33. Zheng, Z., Zhang, M.-J., Zhang, G. \& Chen, G.-Q. Production of 3-hydroxydecanoic acid by recombinant Escherichia coli HB101 harboring phaG gene. Antonie Van Leeuwenhoek 85, 93101 (2004).

34. Goh, E.-B., Baidoo, E. E. K., Keasling, J. D. \& Beller, H. R. Engineering of bacterial methyl ketone synthesis for biofuels. Appl. Environ. Microbiol. 78, 70-80 (2012).

35. Altschul, S. F., Gish, W., Miller, W., Myers, E. W. \& Lipman, D. J. Basic local alignment search tool. J. Mol. Biol. 215, 403-410 (1990).

36. Gerlt, J. A. et al. Enzyme Function Initiative-Enzyme Similarity Tool (EFI-EST): A web tool for generating protein sequence similarity networks. Biochim. Biophys. Acta - Proteins Proteomics 1854, 1019-1037 (2015).

37. Muneer, F. et al. Microbial polyhydroxyalkanoates (PHAs): efficient replacement of synthetic polymers. J. Polym. Environ. 28, 2301-2323 (2020).

38. Schweizer, E. \& Hofmann, J. Microbial type I fatty acid synthases (FAS): major players in a network of cellular FAS systems. Microbiol. Mol. Biol. Rev. 68, 501-517 (2004).

39. He, X.-Y. \& Yang, S.-Y. Histidine-450 Is the catalytic residue of L-3-hydroxyacyl coenzyme A dehydrogenase associated with the large $\alpha$-subunit of the multienzyme complex of 
fatty acid oxidation from Escherichia coli ${ }^{\dagger}$. Biochemistry 35, 9625-9630 (1996).

40. Yang, S. Y. \& Elzinga, M. Association of both enoyl coenzyme A hydratase and 3hydroxyacyl coenzyme A epimerase with an active site in the amino-terminal domain of the multifunctional fatty acid oxidation protein from Escherichia coli. J. Biol. Chem. 268, 6588-92 (1993).

41. Yang, S. Y., Li, J. M., He, X. Y., Cosloy, S. D. \& Schulz, H. Evidence that the fadB gene of the fadAB operon of Escherichia coli encodes 3-hydroxyacyl-coenzyme A (CoA) epimerase, delta 3-cis-delta 2-trans-enoyl-CoA isomerase, and enoyl-CoA hydratase in addition to 3hydroxyacyl-CoA dehydrogenase. J. Bacteriol. 170, 2543-2548 (1988).

42. Smeland, T. E., Cuebas, D. \& Schulz, H. Epimerization of 3-hydroxy-4-trans-decenoyl coenzyme A by a dehydration/hydration mechanism catalyzed by the multienzyme complex of fatty acid oxidation from Escherichia coli. J. Biol. Chem. 266, 23904-23908 (1991).

43. Swarbrick, C. M. D. et al. Mycobacteria encode active and inactive classes of TesB fattyacyl CoA thioesterases revealed through structural and functional analysis. Biochemistry 56, 14601472 (2017).

44. Naggert, J. et al. Cloning, sequencing, and characterization of Escherichia coli thioesterase II. J. Biol. Chem. 266, 11044-50 (1991).

45. McMahon, M. D. \& Prather, K. L. J. Functional screening and in vitro analysis reveal thioesterases with enhanced substrate specificity profiles that improve short-chain fatty acid production in Escherichia coli. Appl. Environ. Microbiol. 80, 1042-1050 (2014).

46. Guevara-Martínez, M. et al. The role of the acyl-CoA thioesterase "YciA" in the production of (R)-3-hydroxybutyrate by recombinant Escherichia coli. Appl. Microbiol. Biotechnol. 103, 3693-3704 (2019).

47. Mendez-Perez, D., Gunasekaran, S., Orler, V. J. \& Pfleger, B. F. A translation-coupling DNA cassette for monitoring protein translation in Escherichia coli. Metab. Eng. 14, 298-305 (2012).

48. Frumkin, I. et al. Codon usage of highly expressed genes affects proteome-wide translation efficiency. Proc. Natl. Acad. Sci. 115, E4940-E4949 (2018).

49. Reis, M. d. Solving the riddle of codon usage preferences: a test for translational selection. Nucleic Acids Res. 32, 5036-5044 (2004).

50. Youngquist, J. T. et al. Production of medium chain length fatty alcohols from glucose in Escherichia coli. Metab. Eng. 20, 177-186 (2013).

51. Nies, S. C. et al. High titer methyl ketone production with tailored Pseudomonas taiwanensis VLB120. Metab. Eng. 62, 84-94 (2020). 


\section{Supplementary Files}

This is a list of supplementary files associated with this preprint. Click to download.

- DNASequenceFiles.zip

- OleoiML1515.xml

- OleoiML1515AddedReactionsandMetabolites.xIsx

- PhaGyieldanalysis.unk

- supplementaryfile.pdf 